\title{
Ductal Carcinoma In situ of the Male Breast
}

\author{
Melissa Brents John Hancock \\ Department of Pathology, University of Mississippi Medical Center, Jackson, MS, USA
}

\section{Established Facts}

- Ductal carcinoma of the male is rare and is usually associated with invasive carcinoma.

- Gynecomastia is known to mask underlying breast calcifications and/or carcinoma.

\section{Novel Insights}

- We report a case of pure high-grade ductal carcinoma in situ of the male breast in a patient presenting with clear nipple discharge whose initial mammography showed benign calcifications.

- Male patients presenting with nipple discharge of any kind should raise the suspicion for breast cancer and prompt further evaluation.

\section{Keywords}

Male breast cancer . Mammography .

Ductal carcinoma in situ

\section{Summary}

Background: Ductal carcinoma in situ of the male breast is an unusual lesion and most often associated with invasive carcinoma. On rare occasions when the in situ component is present in pure form, histological grade is almost always low to intermediate. Imaging for these patients is difficult as gynecomastia is often present and can mask underlying calcifications or carcinoma. Case Report: We report a case of pure high-grade ductal carcinoma in situ of the male breast in a patient with clear nipple discharge. Breast mammography showed bilateral gynecomastia and benign calcifications. Subsequent breast ultrasound showed dilated ducts of the left breast, and a left breast ductogram showed filling defects suggestive of a papilloma. Excisional biopsy and subsequent mastectomy were consistent with high-grade ductal carcinoma in situ. Conclusion: Male breast cancer is uncommon and, although there is increasing awareness, it is less studied compared to female breast cancer. With a clinical history of nipple discharge of any kind, further evaluation with imaging should be considered. In males with gynecomastia, it is important to remember that ductal carcinoma in situ, even of high grade, is difficult to detect on mammography and may not be associated with suspicious calcifications.

(c) 2016 S. Karger GmbH, Freiburg

\section{Introduction}

Ductal carcinoma in situ (DCIS) of the male breast represents approximately $0.1 \%$ of all breast cancers and less than $0.1 \%$ of all cancers in men [1]. Male breast cancer is more frequent in Jewish men and in some African countries, such as Egypt and Zambia. The most prevalent risk factor for male breast cancer is associated

\section{KARGER}

Fax +497614520714

\section{(๑) 2016 S. Karger GmbH, Freiburg}


Fig. 1. (a) Mammogram, left breast gynecomastia. (b) Ultrasound, left breast intraductal dilatation (arrow). (c) Ductogram, left breast intraductal filing defects (arrow).
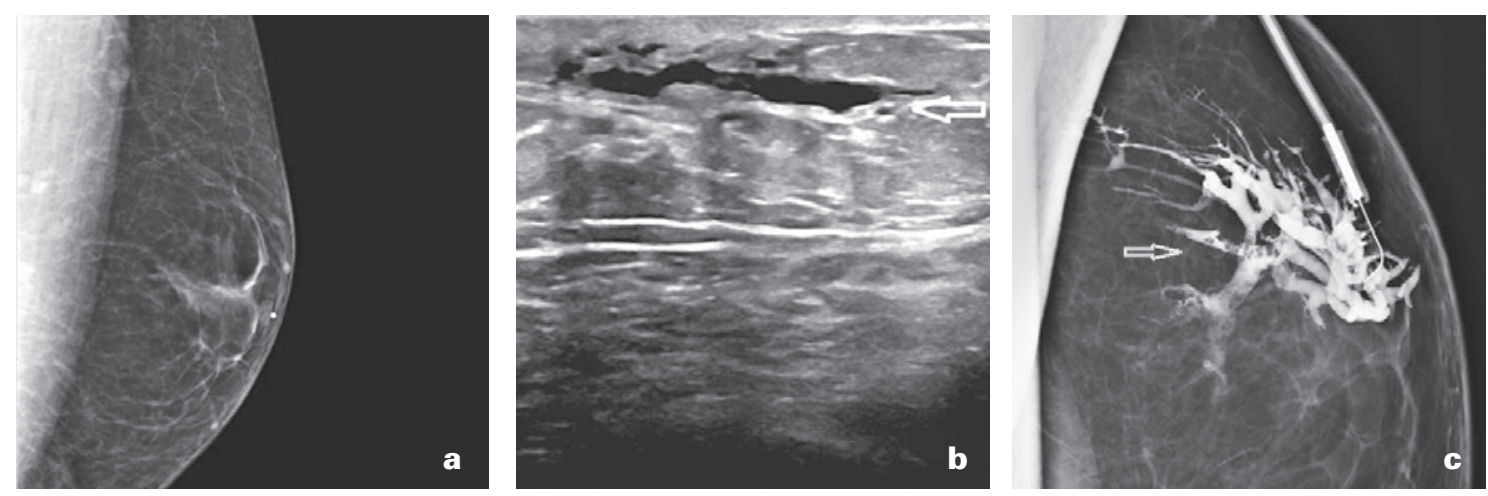

Fig. 2. (a) Papillary ductal carcinoma in situ (DCIS) filling large duct $(\mathrm{H} \& \mathrm{E}, \times 2)$. (b) Grade 3 DCIS with prominent nucleoli, single cell necrosis, and calcification $(\mathrm{H} \& \mathrm{E}, \times 20)$.
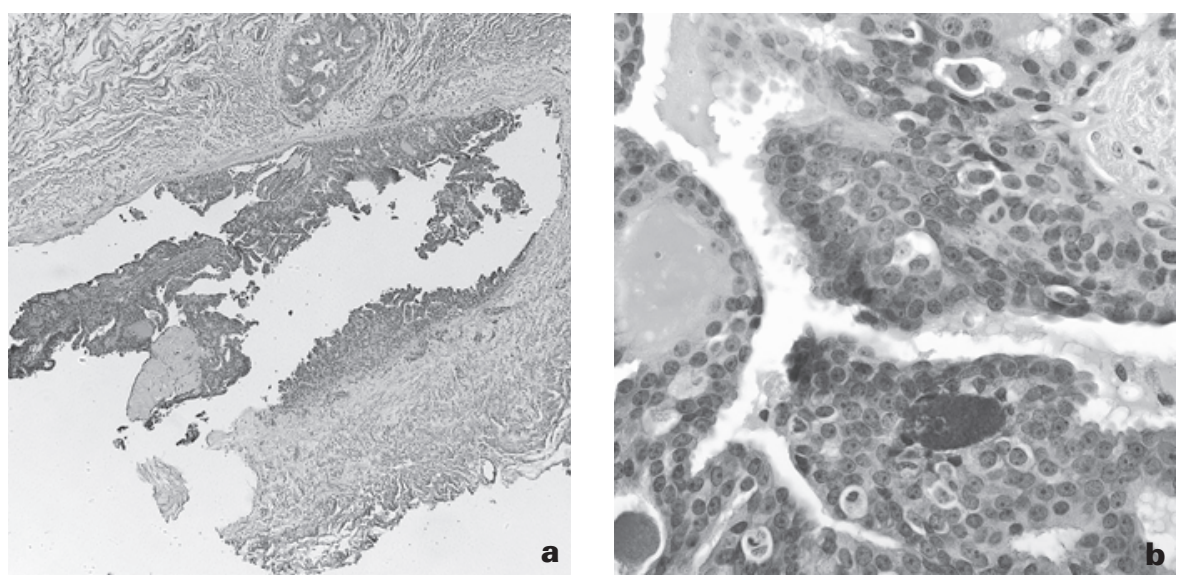

with increasing age and most have been attributed to testicular malfunction and increased estrogen. Other proposed risk factors include family history, mutations of breast cancer genes (BRCA2 > BRCA1), Cowden and Klinefelter syndromes, alcohol consumption, and liver disease. Although claims have been made to the contrary, there is no proven direct link between gynecomastia and male breast cancer [2].

\section{Case Report}

A 58-year-old African American male reported a 6-month history of painful breast masses bilaterally, and with application of external pressure, there was a clear to cloudy left side nipple discharge. There was no history of bloody discharge. Past medical history was pertinent for sickle cell trait, hypertension, and obesity class III (BMI 41.3). Family history was remarkable for a sister with breast cancer and a brother with prostate cancer. Social history indicated occasional use of alcohol but was negative for use of tobacco products.

On physical examination, he was an overweight African American male with symmetrical breasts. On palpitation, there was an ill-defined, mobile, $4 \times 4$-cm mass noted beneath the left nipple by some examiners, while the mass was not detected by others. With applied pressure, a clear stream of discharge fluid was elicited from the left nipple and was felt to be localized to a single duct.

Mammography showed bilateral gynecomastia, multiple bilateral circumscribed nodules, and a few subareolar benign-appearing calcifications in the left breast (fig. 1a). There were no suspicious microcalcifications. A breast ultrasound performed the same day confirmed the bilateral nodules as intramammary lymph nodes and showed dilated ducts on the left (fig. 1b). This was con- sidered a breast imaging reporting and data system category (BI-RADS) 2, but a ductogram was recommended and performed the same day. The ductogram showed multiple filling defects spanning at least $2 \mathrm{~cm}$ in the left breast (fig. 1c). This was considered most consistent with a papilloma, but DCIS could not be excluded and excisional biopsy was recommended.

Our laboratory received a specimen designated left subareolar excision at three o'clock position. It comprised unoriented fibrofatty tissue, $5.4 \times 4.7 \times 2.6 \mathrm{~cm}$ in aggregate, which on gross examination revealed no lesions. The tissue was inked and submitted for routine processing.

Microscopic examination showed DCIS, papillary and cribriform patterns, with microcalcifications (fig. 2). Nuclear grade was 3 , and the lesion extended to an unspecified margin. No invasive cancer was present. The specimen was sent for immunohistochemical examination of estrogen (ER) and progesterone (PR) receptors. The technical processes were performed at Clarient Diagnostic Services (Aliso Viejo, CA), and the results interpreted at our institution by image analysis. The tissue was positive for both ER (with $99 \%$ of nuclei staining with strong intensity) and PR (96\% of nuclei staining strongly). Venipuncture samples were subsequently sent to MYRIAD for BRCA1 and 2 analyses and were negative for the mutations.

The patient continued to report bilateral breast discomfort, and after discussing his diagnosis and options, the patient requested bilateral mastectomy with sparing of the right nipple. He also agreed to sentinel node biopsy but declined radiation treatment. Complete excision was performed 2 months after the initial biopsy, and comprised removal of a left axillary sentinel lymph node, right subcutaneous mastectomy and left simple mastectomy. The right subcutaneous mastectomy showed gynecomastia with no appreciable atypia. The left simple mastectomy showed a residual focus of DCIS measuring $0.6 \mathrm{~cm}$ with negative surgical margins. The architectural pattern was cribriform, with single cell necrosis and apocrine differentiation, and grade 3 nuclei.

The patient was noted to be doing well 4 months postoperatively but was subsequently lost to follow-up at the time of writing. 


\section{Discussion}

The median duration of symptoms for pure DCIS is 2 months, whereas for patients with DCIS and associated invasive carcinoma it is 6 months [3]. Presenting symptoms are similar to female breast cancer. The most common presenting sign is a painless mass, but there may be breast pain, nipple discharge, or nipple retraction. Given the anatomy of the male breast, the lesions are usually central and slightly eccentric to the nipple, thus nipple involvement is relatively more common $[2,4]$.

Screening mammography indicates that calcifications are less frequently associated with male breast cancer as compared to female breast cancer. When present, these calcifications often have a benign or nonspecific appearance. To make matters more challenging, gynecomastia is notorious for masking underlying calcifications and/or carcinoma, even when evaluated in retrospect [4].

Tissue analysis shows that the vast majority of male breast cancers are invasive ductal carcinomas. Invasive lobular carcinoma is rare (1\%), presumably due to the lack of lobular development in the male breast [2]. Lobular carcinoma in situ has been reported but usually in association with an invasive lobular carcinoma [5] Male DCIS represents approximately $10 \%$ of all male breast cancers; therefore, in North America, this should have a prevalence of approximately $1 / 1,000,000$ [2]. Of these, pure DCIS only accounts for $5 \%$ of cases, while the remaining DCIS cases are associated with invasive carcinoma. Among female breast cancer patients, 20\% present with pure DCIS [6]. This discrepancy is thought to be partly due to increased awareness and regular breast screening in female patients, which leads to earlier detection and overall better prognosis. Although male breast cancer has gained more recognition over the past decade, male DCIS still tends to present at a later age and is more often associated with invasive cancer [2].

The histological patterns seen in female DCIS are also seen in males but with varying frequency. Hittmair et al. [3] looked at 84 cases of pure DCIS of the male breast and found that the most common histological subtype was papillary carcinoma (74\%) with a superimposed cribriform pattern. Pure DCIS cases in this series
Table 1. Ductal carcinoma in situ nuclear grade stratification

\begin{tabular}{|c|c|c|}
\hline & Low (NG 1) & High (NG 3) \\
\hline Appearance & $\begin{array}{l}\text { monotonous, lack pleo- } \\
\text { morphism }\end{array}$ & markedly pleomorphic \\
\hline Size & $1.5-2.0 \mathrm{RBC}$ & $>2.5 \mathrm{RBC}$ \\
\hline Features & $\begin{array}{l}\text { chromatin fine; occasional } \\
\text { nucleoli and mitotic figure; } \\
\text { polarization of cells }\end{array}$ & $\begin{array}{l}\text { vesicular; chromatin irregular; } \\
\text { multiple nucleoli; mitoses may } \\
\text { be frequent; loss of cell polarity }\end{array}$ \\
\hline
\end{tabular}

were found to be of either low or intermediate grades. There were no cases of pure high-grade DCIS as seen in our case. The same study also looked at 30 cases of male DCIS with associated invasive breast carcinoma and found $17 \%, 60 \%$, and $23 \%$ of the in situ component to be low, intermediate, and high grades, respectively. Although nuclear grading of DCIS is somewhat subjective, the recommendations made at the international consensus conference in Philadelphia are widely accepted and are summarized in table 1 [7].

Overall, we feel that this was a very challenging case. Given our patient's relatively benign presentation, it was fortunate that prompt follow up with ultrasound and ductogram was performed in a relatively short time span and that the patient was not lost to follow-up. The fact that his symptoms lasted for 6 months in combination with a pathologically high-grade in situ component makes it very likely that the disease would have progressed to invasive carcinoma. In conclusion, our case is unusual in that a pure highgrade DCIS in a male patient was detected prior to progression to invasion. Breast cancer should be considered for any male patient presenting with nipple discharge of any kind and prompt further evaluation.

\section{Disclosure Statement}

The authors declare that they have no competing interests.

\section{References}

1 Romics L, O'Brien ME, Relihan N, et al.: Intracystic papillary carcinoma in a male as a rare presentation of breast cancer: A case report and literature review. J Med Case Rep 2009;3:13.

2 Fentiman I: Male breast cancer: A review. ecancermedicalscience 2009;3:140.
3 Hittmair AP, Lininger RA, Tavassoli FA: Ductal carcinoma in situ (DCIS) in the male breast. A morphologic study of 84 cases of pure DCIS and 30 cases of DCIS associated with invasive carcinoma - a preliminary report. Cancer 1998;83:2139-2149.

4 Evans GF, Anthony T, Turnage RH, et al.: The diagnostic accuracy of mammography in the evaluation of male breast disease. Am J Surg 2001;181:96-100.
5 Erhan Y, Zekioglu O, Erhan Y: Invasive lobular carcinoma of the male breast. Can J Surg 2006;49:365-366.

6 Coroneos CJ, Hamm C: Ductal carcinoma in situ in a 25-year-old man presenting with apparent unilateral gynecomastia. Curr Oncol 2010;17:133-137.

7 The Consensus Conference Committee. Consensus conference on the classification of ductal carcinoma in situ. Cancer 1997;80:1798-1802. 